Pure Mathematical Sciences, Vol. 4, 2015, no. 1, 9 - 15

HIKARI Ltd, www.m-hikari.com

http://dx.doi.org/10.12988/pms.2015.4818

\title{
The k-Fractional Logistic Equation with k-Caputo Derivative
}

\author{
Rubén A. Cerutti \\ Faculty of Exact Sciences \\ National University of the Northeast \\ Av. Libertad 5540 (3400); Corrientes, Argentina
}

Copyright (C) 2014 Rubén A. Cerutti. This is an open access article distributed under the Creative Commons Attribution License, which permits unrestricted use, distribution, and reproduction in any medium, provided the original work is properly cited.

\begin{abstract}
A generalization of the fractional logistic equation by using k-Caputo derivative is introduced. Also a solution that can be expressed en terms of the k-Mittag-Leffler function is obtained.

The development of this paper has been done on the basis what has been done by Camargo and Bruno-Alfonso in [3].
\end{abstract}

Keywords: k-Fractional calculus, k-Mittag-Leffler function, k-RiemannLiouville fractional derivative

\section{Preliminaries}

Generalizations of the classical Gamma function and the Pochhammer's symbol were introduced by Diaz and Pariguan [1].Since then has appeared a significant number of papers extended definitions of fractional order of integrodifferential operators using these generalizations given rise to what may be called the k-Fractional calculus.

Let us remember the definition of the k-Gamma function introduced in [1] and its relations with the classical Gamma function.

Definition 1 Let $z$ be a complex number that $\operatorname{Re}(z)>0$. The $k$-Gamma function is given by the following integral 


$$
\Gamma_{k}(z)=\int_{0}^{\infty} t^{z-1} e^{-\frac{t^{k}}{k}} d t
$$

The relationship between $\Gamma_{k}(z)$ and the classical $\Gamma(z)$ is expressed by

$$
\Gamma_{k}(z)=k^{\frac{z}{k}-1} \Gamma\left(\frac{z}{k}\right) .
$$

It can be seen that $\Gamma_{k}(z)$ is such that $\Gamma_{k}(z) \rightarrow \Gamma(k)$ as $k \rightarrow 1$.

The function $\Gamma_{k}(z)$ satisfies the following properties

1. $\Gamma_{k}(z+k)=z \Gamma_{k}(z)$,

2. $\Gamma_{k}(k)=1$.

For further development of our article, we need to introduce the k-Pochhammer symbol $(\gamma)_{n, k}$ due to Diaz and Pariguan [1] given by the following

Definition 2 Let $\gamma$ be a complex number, $k>0$ and $n \in \mathbb{N}$. The $k$-Pochhammer symbol $(\gamma)_{n, k}$ is

$$
(\gamma)_{n, k}=\gamma(\gamma+k)(\gamma+2 k) \ldots(\gamma+(n-1) k)
$$

It can be seen that $(\gamma)_{n, 1}=(\gamma)_{n}$, the classical Pochhammer symbol. Also verifies the following properties

1. $(\gamma)_{n, k}=k^{n}\left(\frac{\gamma}{k}\right)_{n}$

2. $(\gamma)_{n, k}=\frac{\Gamma_{k}(\gamma+n k)}{\Gamma_{k}(\gamma)}$.

According [2], now we introduce the k-Mittag-Leffler function $E_{k, \alpha, \beta}^{\gamma}(z)$ by the following

Definition 3 Let $k \in \mathbb{R}^{+}, \alpha, \beta, \gamma \in \mathbb{C}, \operatorname{Re}(\alpha)>0, \operatorname{Re}(\beta)>0$.

The $k$-Mittag-Leffler function $E_{k, \alpha, \beta}^{\gamma}(z)$ is given by the following series

$$
E_{k, \alpha, \beta}^{\gamma}(z)=\sum_{n=0}^{\infty} \frac{(\gamma)_{n, k} z^{n}}{\Gamma_{k}(\alpha n+\beta) n !}
$$

where $(\gamma)_{n, k}$ is the $k$-Pochhammer symbol given in (I.3) and $\Gamma_{k}(z)$ is the k-gamma function given in (I.1). 
When we consider $k=\gamma=1$, (I.6) reduce to the classical two parameters Mittag-Leffler function

$$
E_{1, \alpha, \beta}^{1}(z)=\sum_{n=0}^{\infty} \frac{z^{n}}{\Gamma(n \alpha+\beta)}=E_{\alpha, \beta}(z)
$$

and, if $\alpha=\beta=\gamma=k=1$, it result the exponential function

$$
E_{1,1,1}^{1}(z)=e^{z}
$$

The Laplace transform of a function closely related to the k-Mittag-Leffler function is know. It is the following (cf [2])

$$
\mathcal{L}\left\{z^{\frac{\beta}{k}-1} E_{k, \alpha, \beta}^{\gamma}\left(k^{\frac{\alpha}{k}-1} a z\right)\right\}(s)=\frac{s^{\frac{\alpha}{k} \frac{\gamma}{k}} k^{1-\frac{\beta}{k}}}{s^{\frac{\beta}{k}}\left(s^{\frac{\alpha}{k}}-a\right)^{\frac{\gamma}{k}}} ;
$$

for $\left|a s^{-\frac{\alpha}{k}}\right|<1$.

Analogously to what was done with the Riemann-Liouville fractional derivative, we now introduce a modification of the Caputo derivative, modification that involves the k-Riemann-Liouville singular kernel.

Then, we put, by

Definition 4 Let $f$ be a function at least $n$ times differentiable, $n \in \mathbb{N}, \alpha \in \mathbb{R}$ such that $n-1<\alpha<n$.

The $k$-Caputo fractional derivative of order $\alpha$ of the function $f$ is given by

$$
{ }^{c} D_{k}^{\alpha} f(t)=I_{k}^{n-\alpha} f^{(n)}(t),
$$

where $f^{(n)}(t)=D^{n} f(t)$ is the ordinary derivative, and $I_{k}^{n-\alpha}$ is the $k$-RiemannLiouville integral.

Remembering the definition of the k-Riemann-Liouville singular kernel

$$
J_{\gamma, k}(t)= \begin{cases}\frac{t^{\frac{\gamma}{k}-1}}{k \Gamma_{k}(\gamma)} & \text { if } t \geq 0 \\ 0 & \text { if } t<0\end{cases}
$$

the formula (I.9) may be expressed as a convolution

$$
{ }^{c} D_{k}^{\alpha} f(t)=J_{n-\alpha, k} * f^{(n)}(t) .
$$

The Laplace transform of $J_{\gamma, k}(z)$ is (cf [6])

$$
\mathcal{L}\left[\frac{t^{\frac{\alpha}{k}}}{k \Gamma_{k}(z)}\right](s)=(k s)^{-\frac{\alpha}{k}} .
$$




\section{The k-fractional logistic equation}

After the considerations made by Camargo and Bruno-Alfonso in [3], we present the equation

$$
{ }^{c} D_{k}^{\alpha} f(t)=k^{\frac{\alpha-1}{k}} \lambda[1-f(t)]
$$

Where ${ }^{c} D_{k}^{\alpha}$ denote the k-fractional derivative introduced in (I.9), $0<\alpha<1$. Equivalently, we have

$$
{ }^{c} I_{k}^{\frac{1-\alpha}{k}} f^{\prime}(t)=k^{\frac{\alpha-1}{k}} \lambda(1-f(t))
$$

By applying the Laplace transform to the equation (II.2) and taking into account some of their basic properties and the expression (I.12), from the left hand side, we have

$$
\begin{aligned}
\mathcal{L}\left[J_{1-\alpha, k}\right] \cdot \mathcal{L}\left(f^{\prime}\right) & =(k s)^{-\frac{(1-\alpha)}{k}} \cdot \mathcal{L}\left(f^{\prime}\right)= \\
& =(k s)^{\frac{\alpha-1}{k}}\{s F(s)-f(0)\}
\end{aligned}
$$

where $F(s)=\mathcal{L}(f)$

From the right hand member of (II.1) we have

$$
k^{\frac{\alpha-1}{k}} \mathcal{L}[\lambda(1-f(t))]=k^{\frac{\alpha-1}{k}} \lambda\left(\frac{1}{s}-F(s)\right)
$$

Thus, from (II.3) and (II.4) it results

$$
\begin{gathered}
s^{\frac{\alpha-1}{k}}\{s F(s)-f(0)\}=\lambda\left\{\frac{1}{s}-F(s)\right\}, \\
s^{\frac{\alpha-1+k}{k}} \cdot F(s)-s^{\frac{\alpha-1}{k}} f(0)=\lambda\left\{\frac{1}{s}-F(s)\right\} \\
\left\{s^{\frac{\alpha-1+k}{k}}+\lambda\right\} F(s)=s^{\frac{\alpha-1}{k}} f(0)+\frac{\lambda}{s} \\
F(s)=\frac{s^{\frac{\alpha-1}{k}}}{\left(s^{\frac{\alpha-1+k}{k}}+\lambda\right)} f(0)+\frac{\lambda s^{-1}}{\left(s^{\frac{\alpha-1+k}{k}}+\lambda\right)}
\end{gathered}
$$

Note that if in (I.8), $\gamma=k$ is considered, we have

$$
\mathcal{L}\left\{z^{\frac{\beta}{k}-1} E_{k, \alpha, \beta}^{k}\left(k^{\frac{\alpha}{k}-1} a z^{\alpha}\right)\right\}=\frac{s^{\frac{\alpha}{k}} k^{1-\frac{\beta}{k}}}{s^{\frac{\beta}{k}}\left(s^{\frac{\alpha}{k}}-a\right)}
$$


and, taking into account that $(k)_{n, k}=k^{n} n$ !, the left hand member of (II.6) is

$$
\begin{array}{r}
\mathcal{L}\left\{z^{\frac{\beta}{k}-1} \sum_{n=0}^{\infty} \frac{k^{n}\left(k^{\frac{\alpha}{k}-1} a z^{\alpha}\right)^{n}}{\Gamma_{k}(\alpha n+\beta)}\right\}= \\
\mathcal{L}\left\{z^{\frac{\beta}{k}-1} E_{k, \alpha, \beta}\left(k^{\frac{\alpha}{k}} a z^{\alpha}\right)\right\}
\end{array}
$$

Let $\frac{s^{\frac{\alpha-1}{k}}}{\left(s^{\frac{\alpha-1+k}{k}}+\lambda\right)}$; and consider

$$
\begin{aligned}
\frac{s^{\frac{\alpha-1}{k}+1-1}}{\left(s^{\frac{\alpha-1+k}{k}}+\lambda\right)} & =\frac{s^{\frac{\alpha-1+k}{k}} s^{-1}}{\left(s^{\frac{\alpha-1+k}{k}}+\lambda\right)}=\frac{s^{\frac{\alpha-1+k}{k}}}{s\left(s^{\frac{\alpha-1+k}{k}}+\lambda\right)} \\
& =\mathcal{L}\left[E_{k, \frac{\alpha-1+k}{k}}\left(-k^{\frac{\alpha-1+k}{k^{2}}-1} \lambda t^{\frac{\alpha-1+k}{k}}\right)\right]
\end{aligned}
$$

And

$$
\begin{gathered}
\frac{s^{-1}}{\left(s^{\frac{\alpha-1+k}{k}}+\lambda\right)}=\frac{s^{\frac{\alpha-1+k}{k}+\left(-1-\frac{\alpha-1+k}{k}\right)}}{\left(s^{\frac{\alpha-1+k}{k}}+\lambda\right)}=\frac{s^{\frac{\alpha-1+k}{k}}}{s^{1+\frac{\alpha-1+k}{k}}\left(s^{\frac{\alpha-1+k}{k}}+\lambda\right)} \\
=\mathcal{L}\left\{t^{1+\frac{\alpha-1+k}{k}-1} E_{k, \frac{\alpha-1+k}{k}, 1+\frac{\alpha-1+k}{k}}\left[-k^{\frac{\alpha-1+k}{k^{2}}-1} \lambda t^{\frac{\alpha-1+k}{k}}\right]\right\}
\end{gathered}
$$

From (II.5), (II.8) and (II.9) we have

$$
\begin{array}{r}
F(s)=f(0) \mathcal{L}\left(t^{\frac{\alpha-1+k}{k}} E_{k, \frac{\alpha-1+k}{k}, 1+\frac{\alpha-1+k}{k}}\left[-k^{\frac{\alpha-1+k}{k^{2}}-1} \lambda t^{\frac{\alpha-1+k}{k}}\right]\right)+ \\
+\lambda \mathcal{L}\left(E_{k, \frac{\alpha-1+k}{k}}\left[-k^{\frac{\alpha-1+k}{k^{2}}} \lambda t^{\frac{\alpha-1+k}{k}}\right]\right)
\end{array}
$$

and, by applying the inverse Laplace transform it results

$$
\begin{array}{r}
f(t)=\mathcal{L}^{-1}\{F(s)\}=f(0) \cdot E_{k, \frac{\alpha-1+k}{k}}\left[-k^{\frac{\alpha-1+k}{k^{2}}} \lambda t^{\frac{\alpha-1+k}{k}}\right]+ \\
+\lambda t^{\frac{\alpha-1+k}{k}} E_{k, \frac{\alpha-1+k}{k}, 1+\frac{\alpha-1+k}{k}}\left[-k^{\frac{\alpha-1+k}{k^{2}}} \lambda t^{\frac{\alpha-1+k}{k}}\right]
\end{array}
$$

It can be seen that, when $k=1$, from (II.11), we have

$$
f(t)=f(0) E_{\alpha}\left(-\lambda t^{\alpha}\right)+\lambda t^{\alpha} E_{\alpha, \alpha+1}\left(-\lambda t^{\alpha}\right)
$$


which is the result given in [3].

We can see that

$$
\begin{gathered}
E_{k, \frac{\alpha-1+k}{k}, \frac{\alpha-1+k}{k}+1}\left(-(k t)^{\frac{\alpha-1+k}{k}} \lambda\right) \text { it can be written as } \\
E_{k, \frac{\alpha-1+k}{k}, \frac{\alpha-1+k}{k}+1}\left(-(k t)^{\frac{\alpha-1+k}{k}} \lambda\right)= \\
=-\frac{1}{\lambda(k t)^{\frac{\alpha-1+k}{k}}} \sum_{n=0}^{\infty} \frac{\left(-(k t)^{\frac{\alpha-1+k}{k}} \lambda\right)^{n+1}}{\Gamma_{k}\left((n+1)\left(\frac{\alpha-1+k}{k}\right)+1\right)}= \\
=-\frac{1}{\lambda(k t)^{\frac{\alpha-1+k}{k}}}\left\{-\frac{1}{\Gamma_{k}(1)}+\sum_{n=0}^{\infty} \frac{\left(-(k t)^{\frac{\alpha-1+k}{k}} \lambda\right)^{n}}{\Gamma_{k}\left((n+1) \frac{\alpha-1+k}{k}+1\right)}\right\}= \\
=-\frac{1}{\lambda(k t)^{\frac{\alpha-1+k}{k}}}\left\{-\frac{1}{\Gamma_{k}(1)}+E_{k, \frac{\alpha-1+k}{k}}\left(-\lambda(k t)^{\frac{\alpha-1+k}{k}}\right)\right\} .
\end{gathered}
$$

Then, replacing (II.13) in (II.11) it results

$f(t)=f(0) E_{k, \frac{\alpha-1+k}{k}}\left(-\lambda(k t)^{\frac{\alpha-1+k}{k}}\right)+\frac{1}{k^{\frac{\alpha-1+k}{k}}}\left\{-\frac{1}{\Gamma_{k}(1)}+E_{k, \frac{\alpha-1+k}{k}}\left(-\lambda(k t)^{\frac{\alpha-1+k}{k}}\right)\right\}$

or equivalently

$$
f(t)=E_{k, \frac{\alpha-1+k}{k}}\left(-\lambda(k t)^{\frac{\alpha-1+k}{k}}\right)\left\{f(0)-\frac{1}{k^{\frac{\alpha-1+k}{k}}}\right\}+\frac{1}{k^{\frac{\alpha-1+k}{k}}} \cdot \frac{1}{\Gamma_{k}(1)}
$$

Taking $k=1$, it results

$$
f(t)=E_{\alpha}\left(\lambda(-t)^{\alpha}\right)\{f(0)-1\}+1
$$

when $\lambda=1$, coincides with the results due to Figueiredo Camargo and BrunoAlfonso [3]

\section{References}

[1] Diaz, R.; Pariguan, E. On Hypergeometric Functions and k-Pochhammer symbol. Divulgaciones Matemáticas. Vol. 15. 2 (2007)

[2] Dorrego, G.; Cerutti, R. The k-Mittag-Leffler Function. Int. J. Contemp. Math. Sciences. N ${ }^{\circ}$ 15. Vol. 7 (2012).

[3] Figueiredo Camargo, R.; Bruno-Alfonso, A. Equacao Logistica Fracionaria. Anais do Congreso de Matematica Aplicada e Computacional. CMAC Nordeste. Brasil. (2012). 
[4] Haubold, M.; Mathai, A.; Saxena, R. Mittag-Leffler Functions and applications. Journal of Applied Math. Vol 2011. (2011). DOI/10.1155/2011/298628.

[5] Hilfer, R. Threefold Introduccion to fractional derivatives. Anomalous transport: Foundation and applications. (2008)

[6] Romero, L.; Cerutti, R.; Dorrego, G. k-Weyl fractional integral. Int. Journal of Math. Analysis. Vol 6. N 34 (2012), 1685-1691.

Received: August 1, 2014 